\title{
Premalignant changes associated chronic gastritis in Karbala Province: multi institutional study
}

\author{
Rasha Abd Alraouf Neama AlSafi, Mohammed F. Alqanbar, and Wafaa Redha Mohammed Al-Sabbagh
}

Department of Pathology, College of Medicine, Karbala University, Karbala, Iraq.

Correspondence to Rasha Abd Alraouf Neama AlSafi (email: rasha.alsafi78@gmail.com).

(Submitted: 26 April 2019 - Revised version received: 18 May 2019 - Accepted: 13 July 2019 - Published online: 26 October 2019)

\begin{abstract}
Objectives The aim of this study was to investigate the prevalence of gastric premalignant changes as gastric atrophy, intestinal metaplasia and dysplastic changes in patients underwent esophagogastroduodenoscopy in three health institutions in Karbala province.

Methods The data was collected from patients attended the endoscopy units during a period from January to September 2018 in three medical centers in holy Karbala, Iraq, Al Hussein Teaching Hospital, Imam Zain Al Abedin Hospital and Imam AL Hujjah Hospital. It includes 215 males and 285 females who were subjected to esophagogastroduodenoscopy (EGD) from whom mucosal biopsies were taken. These biopsies were stained by Giemsa to detect Helicobacter pylori organisms.

Results The mean age of patients was (40.9 \pm 15 years SD). Helicobacter pylori infection was demonstrated in (71\%) patients, (19.4\%) were presented with glandular atrophy, (7.4\%) out of 500 patients had intestinal metaplasia and only six (1.2\%) patients had dysplastic changes. there was a significant statistical relation between gastric glandular atrophy and age ( $P$-value $=0.03$ ). There was a significant statistical relation between glandular atrophy and infection with $\mathrm{H}$. pylori $(P$-value $=0.001)$. There was a significant negative relation between $\mathrm{H}$. pylori infection and the existence of intestinal metaplasia $(P$-value $=0.0001)$. This study show a significant statistical relationship between the existence of intestinal metaplasia and the occurrence of dysplastic changes $(P$-value $=0.001)$.

Conclusion The current study show a significant relation between aging and the existence of gastric glandular atrophy. There was a significant relation between each of gastric glandular atrophy and intestinal metaplastic changes with the $H$. pylori infection. There was a significant relation between gastric intestinal metaplasia and dysplastic changes.

Keywords premalignant changes, age, H. pylori, gastric atrophy, intestinal metaplasia, dysplasia
\end{abstract}

\section{Introduction}

The incidence rate of gastric cancer has been decreased gradually and continuously over the last years; however it ranks as the fourth most prevalent cancer and a significant leader to cancer related death over the world. ${ }^{1,2}$ Cancer of the stomach from histopathological aspect is of two types, the intestinal and the diffuse types. The occurrence of the intestinal type may be preceded by a series of precancerous pathological lesions and changes such as chronic atrophic gastritis, intestinal metaplasia (IM), and dysplasia. ${ }^{3}$ However, the genesis of the diffuse type is not clearly recognized. ${ }^{4}$

Helicobacter pylori infection is accompanied with a sequential events such as severe gastritis, chronic atrophic gastritis, IM and gastric cancer. ${ }^{5,6}$ intensely atrophied gastric mucosa that associated with intestinal metaplastic changes may be attributed to infection with certain types of high risk $H$. pylori. ${ }^{7,8}$ It was noticed that the infection with $H$. pylori during early childhood resulted in premalignant state of pan-gastritis in adulthood. So, the infection with $H$. pylori earlier during life is considered as an important risk factor that increase the opportunity for the evolution of gastric cancer. ${ }^{9,10}$ Other risk factors of IM are high salt consumption, cigarette smoking, alcohol drinking, and chronic bile reflux. ${ }^{11}$ The prevalence of $H$. pylori infection has been recorded from multiple Middle Eastern countries such as Iraq, Iran, Turkey, Libya, Egypt, Bahrain, Oman, Saudi Arabia, and the United Arab Emirates. It has been found that the rate of infection with $H$. pylori are nearly similar in these countries but still there is little variations in that the prevalence of infection reach its peak in Iran while it is nearly uncommon and rare in Iraq and Egypt. ${ }^{12}$ According to a study conducted at 2009 in Iraq stated that the "The mild pathology and antral-predominant gastritis may help to explain the low cancer rate in Iraq". ${ }^{13}$
The aim of this study was to investigate the prevalence of gastric premalignant changes like gastric atrophy, intestinal metaplasia and dysplastic changes that associated with chronic gastritis and their relation with $H$. pylori infection in different age groups in patients underwent esophagogastroduodenoscopy in three health institutions in Karbala province.

\section{Materials and Methods}

The data was collected from patients attended the endoscopy units during a period from January to September 2018 in three medical centers in holy Karbala, Iraq, Al Hussein Teaching Hospital, Imam Zain Al Abedin Hospital and Imam AL Hujjah Hospital. It includes 500 patients who were subjected to esophagogastroduodenoscopy (EGD) in this period from whom endoscopic mucosal biopsies were taken. Each biopsy contained at least two mucosal pieces that submitted to a conventional histological processing and stained with hematoxylin-eosin stain to assess the presence of gastric mucosal changes. These biopsies were stained by Giemsa to detect $H$. pylori organisms. The collected data include many parameters such as age, sex, and histopathological findings (gastritis, H. pylori infection, atrophic changes, intestinal metaplasia and dysplastic changes). Informed written consent was taken from each patient before the inclusion.

\section{Inclusion Criteria}

All patients who attended hospitals with upper GIT symptoms and underwent EGD with gastric mucosal sampling during the period of the study. 


\section{Exclusion Criteria}

This include patients diagnosed to have gastric cancer, those patients undergoing gastric surgery, patients with upper gastrointestinal bleeding and patients with lacking data.

\section{Statistical Analysis}

Chi-square test was used to measure the association between different parameters and $P$-value of $<0.05$ was considered significant.

\section{Results}

Five hundred patients were subjected to EGD, enrolled in this study during a period from January to September 2018. The mean age of patients was $(40.9 \pm 15$ years standard deviation). There were 215 (43\%) males and 285 (57\%) females. All patients have gastritis $[500(100 \%)] . H$. pylori infection was demonstrated in $355(71 \%)$ patients.

Out of 500 patients, 97 (19.4\%) were presented with glandular atrophy, $37(7.4 \%)$ out of total number of patients had intestinal metaplasia, while only six (1.2\%) patients had dysplastic changes distributed equally between males and females. The results of this study show that the highest frequencies of patients infected with $H$. pylori were distributed between 20 and 59 years of age; however, this relation was not significant statistically $(P$-value $=0.06)$.

There was a significant statistical relation between gastric glandular atrophy and age in that the number of cases with atrophied gastric glands was growing with increasing the age from 20 to 60 years forming about $76(78.3 \%)$ cases $(P$-value $=$ 0.03). Concerning intestinal metaplasia, the results revealed a mounting rate of metaplastic changes with the age and the relationship was statistically near the significant level $(P$-value $=$ 0.052). All cases with dysplasia were six (1.2\%) distributed unevenly through age groups from 30 to 69 years, the relationship of these changes with age was statistically insignificant $(P$-value $=0.7)$.
The distribution and the relationship of the histopathological findings (H. pylori infection, gastric glandular atrophy, intestinal metaplasia and dysplastic changes) with age was demonstrated in Table 1.

Of 355 patients infected with $H$. pylori, there were 88 (24.8\%) cases presented with glandular atrophy in comparison to only $9 / 145(6.2 \%)$ of non-infected patients who presented with atrophied gastric glands and this figure reach a significant level statistically $(P$-value $=0.001)$. Additionally there is a statistical significant negative relationship between intestinal metaplastic changes and the existence of $H$. pylori infection in that 17 patients $(4.8 \%)$ with $H$. pylori infection were presented with intestinal metaplasia in comparison to $20(13.8 \%)$ of non-infected patients presented with intestinal metaplasia on examination as shown in Table 2.

Out of total 37 cases which show intestinal metaplasia there were three $(8.1 \%)$ cases which were diagnosed to have dysplastic changes while three $(0.7 \%)$ cases out of the remaining negative cases show dysplastic changes. This result give a significant statistical relationship between the existence of intestinal metaplasia and the occurrence of dysplastic changes $(P$-value $=0.001)$ as shown in Table 3 .

\section{Discussion}

In the model of gastric carcinogenesis, $H$. pylori has a substantial effect in the development of chronic active gastritis.

Table 2. The relation of $\boldsymbol{H}$. pylori infection with glandular atrophy and intestinal metaplasia

\begin{tabular}{lllllll}
\hline \multirow{2}{*}{$\begin{array}{l}\text { H.pylori } \\
\text { infection }\end{array}$} & \multicolumn{2}{c}{$\begin{array}{c}\text { Atrophy } \\
(\boldsymbol{P} \text {-value }=\mathbf{0 . 0 0 1 )}\end{array}$} & \multicolumn{2}{c}{$\begin{array}{c}\text { Intestinal metaplasia } \\
(\boldsymbol{P} \text {-value }=\mathbf{0 . 0 0 0 1 )}\end{array}$} & \multirow{2}{*}{ Total } \\
\cline { 2 - 5 } & \multicolumn{1}{c}{ Positive (\%) } & Negative (\%) & Positive (\%) & Negative (\%) & \\
\hline Positive & $88(24.8)^{*}$ & $267(75.2)^{*}$ & $17(4.8)^{*}$ & $338(95.2)$ & 355 \\
Negative & $9(6.2)^{* *}$ & $136(93.8)^{* *}$ & $20(13.8)^{* *}$ & $125(86.2)$ & 145 \\
\hline
\end{tabular}

*Percentage out of total positive cases infected with H. pylori.

**Percentage out of total cases not infected with H. pylori.

Table 1. The histopathological findings of $\mathbf{5 0 0}$ patients underwent esophagogastroduodenoscopy and their relations with age

\begin{tabular}{|c|c|c|c|c|c|c|c|c|c|c|}
\hline \multirow{2}{*}{$\begin{array}{l}\text { Histopathological } \\
\text { findings (frequency) }\end{array}$} & \multirow{2}{*}{ Frequencies (\%) } & \multicolumn{8}{|c|}{ Age groups (years) } & \multirow{2}{*}{$P$-value } \\
\hline & & $10-19$ & $20-29$ & $30-39$ & $40-49$ & $50-59$ & $60-69$ & $70-79$ & $\geq 80$ & \\
\hline \multicolumn{11}{|l|}{ H.pylori } \\
\hline - Positive & $355(71)^{*}$ & 29 & 75 & 87 & 73 & 45 & 33 & 13 & 0 & $(0.06)$ \\
\hline - Negative & $145(29)^{*}$ & 5 & 24 & 35 & 34 & 28 & 11 & 6 & 2 & \\
\hline \multicolumn{11}{|l|}{ Glandular atrophy } \\
\hline - Positive & $97(19.4)^{*}$ & 9 & 15 & 19 & 20 & 22 & 5 & 7 & 0 & (0.03) \\
\hline - Negative & $403(80.6)^{*}$ & 25 & 84 & 103 & 87 & 51 & 39 & 12 & 2 & \\
\hline \multicolumn{11}{|l|}{ Intestinal-metaplasia } \\
\hline - Positive & $37(7.4)^{*}$ & 0 & 4 & 10 & 7 & 9 & 5 & 1 & 1 & $(0.052)$ \\
\hline - Negative & $463(92.6)^{*}$ & 34 & 95 & 112 & 100 & 64 & 39 & 18 & 1 & \\
\hline \multicolumn{11}{|l|}{ Dysplastic changes } \\
\hline - Positive & $6(1.2)^{*}$ & 0 & 0 & 2 & 1 & 2 & 1 & 0 & 0 & $(0.7)$ \\
\hline - Negative & $494(98.8)^{*}$ & 34 & 99 & 120 & 106 & 71 & 43 & 19 & 2 & \\
\hline
\end{tabular}

*Percentage from total sample size (500). 
Table 3. The association between intestinal metaplasia and dysplastic changes

\begin{tabular}{lcccc}
\hline \multirow{2}{*}{ Metaplasia } & \multicolumn{2}{c}{ Dysplasia } & \multirow{2}{*}{ Total } & P-value \\
\cline { 2 - 3 } & Negative (\%) & Positive (\%) & & \\
\hline Negative & $460(99.3)^{*}$ & $3(0.7)^{*}$ & 463 & 0.001 \\
Positive & $34(91.9)^{* *}$ & $3(8.1)^{* *}$ & 37 & \\
Total & 494 & 6 & 500 & \\
\hline
\end{tabular}

*The percentage is out of total negative cases.

**The percentage is out of total positive cases.

Chronic H. pylori infection may pave the way to pass through sequential stages of atrophic gastritis, IM, and dysplasia to end with gastric adenocarcinoma. ${ }^{14}$

In this study (according to Table 1$), H$. pylori infection was demonstrated in 355 (71\%) patients and this come in congruent with previous study which give a similar figures. ${ }^{15}$ Other studies reported in the prevalence in North Europe and North America was $<40 \%$, while it was over $70 \%$ in East Asia, Africa, and Middle East region. ${ }^{16,17}$ which comes in consistency with the results of this study while it is far from the figure detected in a Turkish study which found $H$. pylori positivity to be $82 \% .^{18}$

The differences in the reported figures may be related to the point that the incidence actually varies according to living environment, occupation, and geographic region in addition to the diversity in the detection methods. In the current study, $97(19.4 \%)$ patients were presented with glandular atrophy; the prevalence is different from another Iraqi study done in Dohuk, with smaller sample size which record glandular atrophy in just $3 \%$ of gastric biopsies ${ }^{13}$ and may be more similar to a study conducted in Al-Kuwait, in which $28.3 \%$ of patients had atrophic gastritis while the prevalence still high and were $65 \%$ and $54 \%$ of examined Jordanian and Egyptian patients respectively. ${ }^{19-21}$ In a Turkish study, the atrophy was found in $75 \%$ of the subjects ${ }^{22}$ the differences in the results may be attributed to the differences in samples size that affect the results or due to subjectivity between pathologists in making the diagnosis of glandular atrophy. In this study, 37 (7.4\%) out of total number of patients had intestinal metaplasia, this figure was similar to that detected in two other studies in United States and Netherlands in which the prevalence of intestinal metaplasia were $7 \%$ and $8 \%$ respectively. ${ }^{23,24}$ Almouradi et al. ${ }^{25}$ reported that, among the 437 patients who had gastric biopsies performed, 66 were found to have gastric IM and they observed that the overall prevalence was $15 \%$. Additional Turkish study reporting a prevalence of intestinal metaplasia of $13.8 \% .^{3}$

The commonness of gastric intestinal metaplasia publically is still hardly to be ascertain because of the symptomless character of this lesion. There is a wide variation in the prevalence of gastric IM between these studies may be related to the variability in localization and sampling of focal metaplastic lesions. ${ }^{3}$ From 500 cases only six (1.2\%) patients had dysplastic changes. A compatible result was detected by a Turkish study in which dysplastic changes was $2 \%^{18}$ while it differ from that recorded by Iranian study in which the dysplastic changes may reach up to $71 \%$ and higher. ${ }^{26}$ According to the results of this study, there is a significant relationship between the age and glandular atrophy but there was no such a relation with other factors like $H$. pylori infection, intestinal metaplasia and dysplastic changes. This result come in consistency with other study which find that subjects with glandular atrophy were significantly older ${ }^{27}$ while it differs from other Turkish studies which show that intestinal metaplasia and $H$. pylori infection is detected in elderly subjects. ${ }^{18,28}$

This could be attributed to environmental and dietary habits that predispose to early $H$. pylori infection and its consequences, making them unrelated to the age as an influencing factor, while still glandular atrophy considered part of general degenerative process rather than related to $H$. pylori infection.

In this study (according to Table 2), there were 24.8\% cases which were diagnosed to have $H$. pylori infection were presented with glandular atrophy. In two different Turkish studies, histopathological examination reveal that the mucosal atrophy was found in $43-75 \%$ of $H$. pylori infected subjects, ${ }^{22,29}$ In Iran, in a study, the atrophied gastric mucosal epithelium was seen in $22 \%$ of gastric biopsies. ${ }^{26}$ In Al Kuwait, $28.3 \%$ of $H$. pylori infected patients were had atrophic gastritis. ${ }^{19}$ Gastric atrophy was observed in $65 \%$ and $54 \%$ of examined patients in Jordan and Egypt, respectively. ${ }^{20,21}$ Again this could be attributed to subjective criteria in determination of gastric atrophy as well as difference in the sample size and ethnicity. The current study show that there is a significant negative relationship between $H$. pylori infection and gastric intestinal metaplasia in that $4.8 \%$ of patients presented with $H$. pylori infection were diagnosed to have intestinal metaplastic changes in comparison to $13.8 \%$ of cases without $H$. pylori infection show intestinal metaplasia on examination. This figure is similar to that recorded by other studies. ${ }^{8,25,30}$

Intestinal metaplasia may cause lower diagnostic accuracy of $H$. pylori with histologic examination. ${ }^{11}$ As clearly known, $H$. pylori selectively lives in gastric mucosa. Hence, the impairment of $H$. pylori colonization in the areas with intestinal metaplasia is predictable. ${ }^{16}$ At the same time, although the gastric mucosa with intestinal metaplasia looks like the intestinal mucosa, it would still have the features of gastric mucosa according to the grade of metaplasia. ${ }^{18}$

According to the results of this study (Table 3), there was a significant statistical relationship between the existence of intestinal metaplasia and the occurrence of dysplastic changes. This result may be harmonized with many recent studies..$^{31-33}$

\section{Conclusion}

The current study provides information about the prevalence of $H$. pylori infection in patients underwent EGDs in three main institutions in Karbalaa province was $71 \%$. The prevalence of intestinal metaplasia is a premalignant changes recording an alarming figure among patients with chronic gastritis.

It was noticed that prevalence of gastric glandular atrophy was increased gradually with age with no such relation with intestinal metaplasia and dysplastic changes as premalignant changes occur in younger age groups. The absence of significant relation between age and IM could be considered as alarming feature that these changes could be occur earlier in our population.

\section{Conflicts of Interest}

None. 


\section{References}

1. Ferlay J, Shin HR, Bray F, Forman D, Mathers C, Parkin DM. Estimates of worldwide burden of cancer in 2008: GLOBOCAN 2008. Int J Cancer. 2010;127:2893-2917.

2. Ferro A, Peleteiro B, Malvezzi M, Bosetti C, Bertuccio P, Levi F, et al. Worldwide trends in gastric cancer mortality (1980-2011), with predictions to 2015, and incidence by subtype. Eur J Cancer. 2014;50:1330-1344.

3. Olmez S, Aslan M, Erten R, Sayar S, Bayram I. The prevalence of gastric intestinal metaplasia and distribution of helicobacter pylori infection, atrophy, dysplasia, and cancer in its subtypes. Gastroenterol Res Pract. 2015;2015:434039

4. Park DY, Srivastava A, Kim GH, Mino-Kenudson M, Deshpande V, Zukerberg LR, et al. CDX2 expression in the intestinal-type gastric epithelial neoplasia: frequency and significance. Mod Pathol. 2010;23:54-61.

5. Houghton J, Wang TC. Helicobacter pylori and gastric cancer: a new paradigm for inflammation-associated epithelial cancers. Gastroenterology. 2005:128:1567-1578. DOl: https://doi.org/10.1053/j.gastro.2005.03.037

6. Catalano V, Labianca R, Beretta GD, Gatta G, de Braud F, van Cutsem E. Gastric cancer. Crit Rev Oncol Hematol. 2009:71:127-164

7. Uemura N, Okamoto S, Yamamoto S, Matsumura N, Yamaguchi S, Yamakido M, et al. Helicobacter pylori infection and the development of gastric cancer. N Engl J Med. 2001:345:784-789.

8. Lee YC, Chen TH, Chiu HM, Shun CT, Chiang H, Liu TY, et al. The benefit of mass eradication of Helicobacter pylori infection: a community-based study of gastric cancer prevention. Gut. 2013;62:676-682

9. Blaser MJ, Nomura A, Lee J, Stemmerman GN, Perez-Perez Gl. Early-life family structure and microbially induced cancer risk. PLoS Med. 2007;4:e7.

10. Dinis-Ribeiro M, Areia M, de Vries AC, Marcos-Pinto R, Monteiro-Soares M, O'Connor A, et al. Management of precancerous conditions and lesions in the stomach (MAPS): guideline from the European Society of Gastrointestinal Endoscopy (ESGE), European Helicobacter Study Group (EHSG), European Society of Pathology (ESP), and the Sociedade Portuguesa de Endoscopia Digestiva (SPED). Endoscopy. 2012;44:74-94.

11. Correa P, Piazuelo MB, Wilson KT. Pathology of gastric intestinal metaplasia: clinical implications. Am J Gastroenterol. 2010;105:493-498.

12. GLOBOCAN IARC. Cancer map: male stomach cancer, age-standardized incidence rate per 100,000 in 2002. Available from: URL: http://www-dep. iarc.fr/ (Accessed 30 May 2007).

13. Hussein NR, Napaki SM, Atherton JC. A study of Helicobacter pyloriassociated gastritis patterns in Iraq and their association with strain virulence. Saudi J Gastroenterol. 2009;15:125-127.

14. Fuccio L, Zagari RM, Minardi ME, Bazzoli F. Systematic review: Helicobacter pylori eradication for the prevention of gastric cancer. Aliment Pharmacol Ther. 2007:25:133-141.

15. Yasir S, Moin F, Akhtar SM. Frequency of helicobacter pylori infection on histopathology in patients with dyspepsia. Am J Clin Med Res. 2014;2 53-56.

16. Ahn HJ, Lee DS. Helicobacter pylori in gastric carcinogenesis. World J Gastrointest Oncol. 2015;7:455-465.

17. Eusebi LH, Zagari RM and Bazzoli F. Epidemiology of helicobacter pylori infection. Helicobacter. 2014;19:1-5.

18. Ozakay A, Kınacı E, Bayrak S, Pasaoğlu E, Sevinc MM, Dursun N. Helicobacter pylori, intestinal metaplasia, and the accuracy of biopsies in metaplastic gastric mucosa. Int J Clin Exp Med. 2017;10:5332-5337.
19. Sarkar C, Anim JT, Ibrahim BH. Atrophic gastritis and intestinal metaplasia in Helicobacter pylori-associated antral gastritis. Med Princ Pract. 1994;4: 197-203.

20. Korstanje A, den Hartog G, Biemond I, Lamers CB. The serological gastric biopsy: a non-endoscopical diagnostic approach in management of the dyspeptic patient significance for primary care based on a survey of the literature. Scand I Gastroenterol Suppl. 2002:37:22-26.

21. Matalka II, Al-Omari FA, Al-Jarrah MA, Obeidat FN, Kanaan FM. Image-based discriminating morphological features for gastric atrophy assessment: a step to go further. Pathol Res Pract. 2008;204:235-240.

22. Kaklikkaya N, Cubukcu K, Aydin F, Bakir T, Erkul S, Tosun I, et al. Significance of cagA status and vacA subtypes of Helicobacter pylori in determining gastric histopathology: virulence markers of H. pylori and histopathology. J Gastroenterol Hepatol. 2006:21:1042-1047.

23. Sonnenberg A, Lash RH, Genta RM. A national study of Helicobactor pylori infection in gastric biopsy specimens. Gastroenterology. 2010;139:1894-1901.e2; quiz e12.

24. de Vries AC, van Grieken NC, Looman CW, Casparie MK, de Vries E, Meijer GA, et al. Gastric cancer risk in patients with premalignant gastric lesions: a nationwide cohort study in the Netherlands. Gastroenterology. 2008:134:945-952.

25. Almouradi T, Hiatt T, Attar B. Gastric intestinal metaplasia in an underserved population in the USA: prevalence, epidemiologic and clinical features. Gastroenterol Res Pract. 2013;2013:856256.

26. Malekzadeh R, Sotoudeh M, Derakhshan MH, Mikaeli J, Yazdanbod A Merat S, et al. Prevalence of gastric precancerous lesions in Ardabil, a high incidence province for gastric adenocarcinoma in the northwest of Iran. J Clin Pathol. 2004;57:37-42.

27. den Hoed CM, van Eijck BC, Capelle LG, van Dekken H, Biermann K, Siersema $P D$, Kuipers EJ, et al. The prevalence of premalignant gastric lesions in asymptomatic patients: predicting the future incidence of gastric cancer Eur J Cancer. 2011:47:1211-1218.

28. Çınar A, Sadıç M, Atılgan Hï, Baskın A, Koca G, Demirel K, Korkmaz M. Prevalence of Helicobacter pylori infection in school and pre-school aged children with C-14 urea breath test and the association with familial and environmental factors. Mol Imaging Radionucl Ther. 2015;24:66-70.

29. Fikret D, Kaya Ö, Suna E, Vahap O, Mustafa A, Sebnem A. Relationship between atrophic gastritis, intestinal metaplasia, dysplasia and Helicobacter pylori infection. Turk J Gastroenterol. 2001;12:169-170.

30. Galiatsatos P, Wyse J, Szilagyi A. Accuracy of biopsies for Helicobacter pylori in the presence of intestinal metaplasia of the stomach. Turk J Gastroenterol. 2014;25:19-23

31. Gomez JM, Patrie JT, Bleibel W, Frye JW, Sauer BG, Shami VM, et al. Gastric intestinal metaplasia is associated with gastric dysplasia but is inversely correlated with esophageal dysplasia. World J Gastrointest Endosc. 2017;9:61-69.

32. Bozorgnia MA, Kashfi SMH, Ariana M, Ghalkhani F, Iravani S, Lashkari MH, et al. Prevalence and correlation of chronic atrophic gastritis, intestinal metaplasia and other precancerous lesions of stomach in Iran: a historical cohort study. Transl Gastrointest Cancer. 2015:4:413-422

33. Chon I, Choi C, Shin CM, Park YS, Kim N, Lee DH. Effect of Helicobacter pylori eradication on subsequent dysplasia development after endoscopic resection of gastric dysplasia. Korean J Gastroenterol. 2013;61:307-312.

This work is licensed under a Creative Commons Attribution-NonCommercial 3.0 Unported License which allows users to read, copy, distribute and make derivative works for non-commercial purposes from the material, as long as the author of the original work is cited properly. 